\title{
Risk Stratification for Patients with Chest Pain Discharged Home from the Emergency Department
}

\author{
Peter A. Kavsak ${ }^{1, *}$, Joshua O. Cerasuolo ${ }^{2}$, Shawn E. Mondoux ${ }^{3}$, Jonathan Sherbino ${ }^{3}$, Jinhui Ma 4 , \\ Brock K. Hoard ${ }^{5}$, Richard Perez ${ }^{2}$, Hsien Seow ${ }^{2}$, Dennis T. Ko ${ }^{6}$ and Andrew Worster ${ }^{3}$ \\ 1 Department of Pathology and Molecular Medicine, McMaster University and Juravinski Hospital and \\ Cancer Centre, 711 Concession Street Hamilton, Hamilton, ON L8V 1C3, Canada \\ 2 ICES McMaster, Faculty of Health Sciences, McMaster University, Hamilton, ON L8S 4K1, Canada; \\ Joshua.Cerasuolo@ices.on.ca (J.O.C.); richard.perez@ices.on.ca (R.P.); seowh@mcmaster.ca (H.S.) \\ 3 Division of Emergency Medicine, McMaster University, Hamilton, ON L8N 3Z5, Canada; \\ shawn.e.mondoux@gmail.com (S.E.M.); sherbino@gmail.com (J.S.); worstea@mcmaster.ca (A.W.) \\ 4 Department of Health Research Methods, Evidence and Impact, McMaster University, Hamilton, \\ ON L8N 3Z5, Canada; maj26@mcmaster.ca \\ 5 McMaster University, Hamilton, ON L8N 3Z5, Canada; hoardb@mcmaster.ca \\ 6 ICES Central, Toronto, ON M4N 3M5, Canada; dennis.ko@ices.on.ca \\ * Correspondence: kavsakp@mcmaster.ca; Tel.: +905-521-2100
}

Received: 13 August 2020; Accepted: 10 September 2020; Published: 12 September 2020

\begin{abstract}
For patients with chest pain who are deemed clinically to be low risk and discharged home from the emergency department (ED), it is unclear whether further laboratory tests can improve risk stratification. Here, we investigated the utility of a clinical chemistry score (CCS), which comprises plasma glucose, the estimated glomerular filtration rate, and high-sensitivity cardiac troponin (I or $\mathrm{T})$ to generate a common score for risk stratification. In a cohort of 14,676 chest pain patients in the province of Ontario, Canada and who were discharged home from the ED (November 2012-February 2013 and April 2013-September 2015) we evaluated the CCS as a risk stratification tool for all-cause mortality, plus hospitalization for myocardial infarction or unstable angina (primary outcome) at 30,90 , and 365 days post-discharge using Cox proportional hazard models. At 30 days the primary outcome occurred in $0.3 \%$ of patients with a CCS $<2(n=6404), 0.9 \%$ of patients with a CCS $=2$ $(n=4336)$, and $2.3 \%$ of patients with a CCS $>2(n=3936)(p<0.001)$. At 90 days, patients with CCS $<2$ (median age $=52 y(I Q R=46-60), 59.4 \%$ female) had an adjusted HR $=0.51$ (95\% confidence interval $(\mathrm{CI})=0.32-0.82$ ) for the composite outcome and patients with a CCS $>2$ (median age $=74 \mathrm{y}$ $(\mathrm{IQR}=64-82), 48.0 \%$ female $)$ had an adjusted $\mathrm{HR}=2.80(95 \% \mathrm{CI}=1.98-3.97)$. At 365 days, $1.3 \%, 3.4 \%$, and $11.1 \%$ of patients with a CCS $<2,2$, or $>2$ respectively, had the composite outcome $(p<0.001)$. In conclusion, the CCS can risk stratify chest pain patients discharged home from the ED and identifies both low- and high-risk patients who may warrant different medical care.
\end{abstract}

Keywords: clinical chemistry score; high-sensitivity cardiac troponin; chest pain; emergency department; discharged; risk stratification

\section{Introduction}

Now that high-sensitivity cardiac troponin (hs-cTn) assays are globally available and are considered the gold standard test for detecting myocardial injury, the utility of other cardiac biomarkers for investigating patients with chest pain in the emergency department (ED) has diminished [1-6]. In this regard, there has been much interest in applying different high-sensitivity cardiac troponin I (hs-cTnI) or high-sensitivity cardiac troponin $\mathrm{T}$ (hs-cTnT) concentration cutoffs for risk stratification [6-8]. However, a limitation of this approach, is that it relies solely on the measurement of a single analyte 
(hs-cTnI or hs-cTnT), and as such is subject to the well-documented analytical issues of these assays affecting the accuracy and reproducibility of the results [2,9-11]. Moreover, there are other common biomarkers (e.g., glucose and creatinine), besides $\mathrm{cTn}$, that have important pathophysiological roles in acute coronary syndrome (ACS) and might influence risk stratification [12-14].

We have previously derived and validated a simple clinical chemistry score (CCS) at ED presentation that has superior diagnostic performance than either hs-cTnI or hs-cTnT alone for myocardial infarction (MI) [15]. Briefly, the CCS is a six-category score (0 to 5) with points derived from different levels of glucose, the estimated glomerular filtration rate (eGFR), and hs-cTnI or hs-cTnT using established cutoffs [15]. The diagnostic performance of the CCS has been validated in different settings and using different manufacturers' assays [16-18]. The objective for the present study was to assess whether the CCS would provide additional risk stratification information for chest pain patients discharged home from the ED, which are overall a very low risk group of individuals with a reported composite outcome of all-cause death, MI or unstable angina (UA) at 30 days being $\leq 1.0 \%$ in the province of Ontario, Canada [19].

\section{Methods}

\subsection{Study Design and Population}

The study population of 14,676 ED patients with chest pain was comprised of two different cohorts: cohort 1 included 1367 patients who underwent hs-cTnI testing (Abbott Diagnostics) and cohort 2 included 13,309 patients who underwent hs-cTnT testing (Roche Diagnostics) (see Figure 1 for flow diagram). For cohort 1, consecutive ED patients (first presentation from 28 November 2012 to 28 February 2013) who were investigated for possible ACS at three hospitals (teaching hospitals) in the city of Hamilton, Ontario, Canada and had cardiac troponin results were selected $(n=6641)$ [20]. Patients were excluded if they did not have glucose results, creatinine results (for eGFR calculation via CKD-EPI equation), Abbott hs-cTnI results, or missing information which prevented linkages to databases $(n=667)$, were not discharged home $(n=3537)$, did not have an electrocardiogram (ECG) conducted $(n=835)$, or were younger than 40 years or older than 105 years $(n=235)$, leaving 1367 patients discharged home from the ED with a diagnosis of chest pain [19-21].

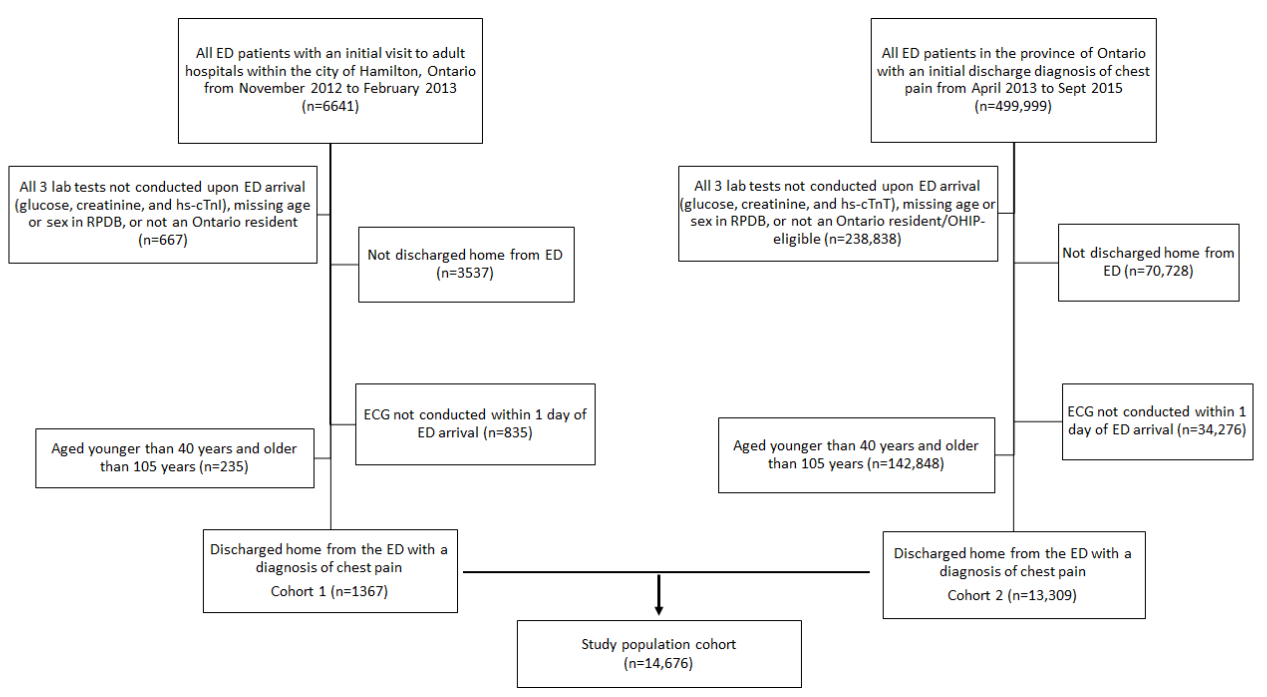

Figure 1. Flow diagram of study population cohort. ED: emergency department; hs-cTn: high-sensitivity cardiac troponin; RPDB: registered persons database; OHIP: Ontario health insurance plan; ECG: electrocardiogram.

For cohort 2, all ED patients (first presentation between April 2013 to September 2015) in the province of Ontario, Canada discharged from the ED with a diagnosis of chest pain $(n=499,999)$ 
were selected [21]. Patients were excluded if they did not have glucose and creatinine results (for eGFR calculation via CKD-EPI equation [22]), Roche hs-cTnT results (laboratory data obtained from the Ontario Laboratory Information System) or had missing information which prevented linkages to databases $(n=238,838)$, were not discharged home $(n=70,728)$, did not have an ECG conducted ( $n=34,276)$, or were younger than 40 years or older than 105 years $(n=142,848)$, leaving 13,309 patients discharged home from the ED ( 3 community hospitals, 1 small hospital and 5 teaching hospitals) with a diagnosis of chest pain $[19,21]$. The final study population consisted of patients from both cohorts of whom had been discharged home, had all necessary information completed, and fell within the correct age range, leaving 14,676 suitable patients. Of note, the age range of 40 to 105 years as well as the requirement for an ECG was to identify a population more likely to have symptoms/pain related to cardiac origin (see Supplement for description of cohort 1 and cohort 2).

\subsection{Clinical Chemistry Score (CCS)}

The CCS consists of the sum of points obtained when using an algorithm that requires the earliest glucose level, eGFR level, and hs-cTnI or hs-cTnT levels for the derivation of the scores [15]. Briefly, the scores are generated for each patient as follows: if glucose level $\geq 5.6 \mathrm{mmol} / \mathrm{L}$, then assign 1 point (if below then no points); if eGFR $<90 \mathrm{~mL} / \mathrm{min} / 1.73 \mathrm{~m}^{2}$, then assign 1 point (if above then no points); if hs-cTnI level $<4 \mathrm{ng} / \mathrm{L}=0$ points or $4-14 \mathrm{ng} / \mathrm{L}=1$ point or $15-30 \mathrm{ng} / \mathrm{L}=2$ points or $>30 \mathrm{ng} / \mathrm{L}=3$ points; or if hs-cTnT level $<8 \mathrm{ng} / \mathrm{L}=0$ points or $8-18 \mathrm{ng} / \mathrm{L}=1$ point or $19-30 \mathrm{ng} / \mathrm{L}=2$ points or $>30 \mathrm{ng} / \mathrm{L}=3$ points [15]. The final CCS was the sum of the points from glucose, eGFR and hs-cTnI or hs-cTnT derived from patients from both cohorts.

\subsection{Outcomes and Statistical Analysis}

The primary outcome was the composite of all-cause death, MI or unstable angina (UA) as previously described [19]. Secondary outcomes included death/MI alone and major adverse cardiac events (MACE; defined as the composite of death/MI/UA/percutaneous coronary intervention $[\mathrm{PCI}]$ or coronary artery bypass grafting [CABG] $[15,21]$. Administrative and clinical databases housed at the Institute for Clinical Evaluative Sciences (ICES, Toronto, Ontario, Canada) via unique encrypted patient identifiers were used to obtain past medical history and outcomes. The Ontario Registered Persons Database (RPDB) contained all information on patient demographics and death date. All inpatient hospital discharges were captured in the Canadian Institute of Health Information (CIHI) Discharge Abstract Database (e.g., MI from diagnostic codes in the hospitalization database which is available for all patients in Ontario). We used the Ontario Health Insurance Plan (OHIP) database to capture all physician billings and outpatient visits. These datasets were linked using unique encoded identifiers and analyzed at ICES. Outcomes were evaluated at 30, 90, and 365 days [19-21]. The analyses were from the patients' earliest record with all subsequent ED visits excluded (i.e., unique patient only represented once in the analysis).

A miss event rate of $1 \%$ for an acute ischemic event/death for discharged ED patients at 30 days is considered by several emergency medicine groups to be acceptable [23,24]. Accordingly, we set the score from the CCS that achieved close to $1 \%$ event rate at 30 days as the referent group. Scores below and above were grouped together to yield three different groups: low CCS, referent, and high CCS. Among these three groups we compared baseline characteristics (demographic and clinical) with a five-year look-back across these categories. Kaplan-Meier survival curves were constructed for the three CCS categories and log-rank test was used to compare the survival difference between these categories for the primary and secondary outcomes. We also performed Cox proportional hazard modeling for the primary outcome (model 1 unadjusted, model 2 adjusted for age and sex and model 3 adjusted for age, sex, past medical history of arrhythmia, heart failure, diabetes, hypertension, MI, peripheral vascular disease, renal disease, stroke and UA) to assess the hazard ratio (HR) of CCS categories with and without adjustment for confounders. For the secondary outcomes model 3 was further adjusted for history of PCI and CABG. The negative predictive values (NPVs) 
were determined for 30 day MACE for CCS $<2$ and hs-cTn $<5 \mathrm{ng} / \mathrm{L}$, as a common cutoff level of $5 \mathrm{ng} / \mathrm{L}$ for both the Roche and Abbott hs-cTn assays has been proposed [25]. NPV estimates $\geq 99.5 \%$ were deemed acceptable [26]. Statistical analyses (e.g., means compared using ANOVA, medians using Kruskal-Wallis test, categorical variables using chi-squared test, Cox modelling, c-statistic, Kaplan-Meier and log-rank analyses) were performed using SAS 9.1.3 software (SAS Institute Inc, Cary, NC, USA) while NPVs were calculated using MedCalc Statistical Software version 19.4.0 (MedCalc Software Ltd., Ostend, Belgium). The Hamilton Integrated Research Ethics Board approved this study (project number: 4717-D, date: March 12, 2018).

\section{Results}

The overall study population cohort $(n=14,676)$ median age (interquartile range; IQR) was 59 years (50 to 71 ) with $55.1 \%$ of the patients being female. Only a third (33\%) of the study population had a follow-up with a cardiologist within 30 days following the ED discharge (Table 1). At 30 days following ED discharge, there were 147 unique patients that had the primary outcome ( $1.0 \%$ of total), with patients with a CCS $=2(n=4336$, median age [IQR] $=62$ years [52 to 71], 55.3\% females) having 37 instances of the composite outcome $(0.9 \%)$ (set as the referent group). Patients with a CCS $<2$ $(n=6404$, median age [IQR] $=52$ years [ 46 to 60$], 59.4 \%$ female) had fewer composite outcomes $(n=18$; $0.3 \%)$ while patients with a CCS $>2(n=3936$, median age [IQR] $=74$ years [64 to 82$], 48 \%$ females $)$ had more outcomes $(n=92,2.3 \%)$ as compared to the CCS $=2$ reference group $(p<0.001)$ (Table 1$)$. The median (IQR) hs-cTnI and hs-cTnT concentrations for the CCS $<2,2$, $>2$ groups were $2 \mathrm{ng} / \mathrm{L}(1-3)$ and $5 \mathrm{ng} / \mathrm{L}$ (3-6), $3 \mathrm{ng} / \mathrm{L}(2-5)$ and $7 \mathrm{ng} / \mathrm{L}$ (5-13), $9 \mathrm{ng} / \mathrm{L}$ (6-16) and $14 \mathrm{ng} / \mathrm{L}$ (12-24), respectively (see Supplemental Tables S1 and S2).

Table 1. Comparison of baseline characteristics and crude outcomes by CCS $(<2,2,>2)$ for patients with a diagnosis of chest pain discharged home from the ED.

\begin{tabular}{|c|c|c|c|c|c|}
\hline Variable & $\mathrm{CCS}<2$ & $\mathrm{CCS}=2$ & $\mathrm{CCS}>2$ & Total & $p$ Value \\
\hline \multicolumn{6}{|c|}{ Demographics } \\
\hline Age in years, Median (IQR) & $52(46-60)$ & $62(52-71)$ & $74(64-82)$ & $59(50-71)$ & $<0.001$ \\
\hline $\operatorname{Sex}(\mathrm{F})$ & $3805(59.4 \%)$ & $2396(55.3 \%)$ & $1890(48.0 \%)$ & $8091(55.1 \%)$ & $<0.001$ \\
\hline \multicolumn{6}{|c|}{ Past medical history, n (\%) } \\
\hline Congestive heart failure & $129(2.0 \%)$ & $226(5.2 \%)$ & $841(21.4 \%)$ & $1196(8.1 \%)$ & $<0.001$ \\
\hline Chronic obstructive pulmonary disorder & $753(11.8 \%)$ & $772(17.8 \%)$ & $1042(26.5 \%)$ & $2567(17.5 \%)$ & $<0.001$ \\
\hline Diabetes & $819(12.8 \%)$ & $967(22.3 \%)$ & $1559(39.6 \%)$ & $3345(22.8 \%)$ & $<0.001$ \\
\hline Hypertension & $2308(36.0 \%)$ & $2472(57.0 \%)$ & $3138(79.7 \%)$ & $7918(54.0 \%)$ & $<0.001$ \\
\hline Myocardial infarction & $171(2.7 \%)$ & $209(4.8 \%)$ & $491(12.5 \%)$ & $871(5.9 \%)$ & $<0.001$ \\
\hline Percutaneous coronary intervention & $190(3.0 \%)$ & $243(5.6 \%)$ & $375(9.5 \%)$ & $808(5.5 \%)$ & $<0.001$ \\
\hline Coronary artery bypass grafting & $28(0.4 \%)$ & $61(1.4 \%)$ & $115(2.9 \%)$ & $204(1.4 \%)$ & $<0.001$ \\
\hline Echocardiography & $2251(35.1 \%)$ & $2043(47.1 \%)$ & $2577(65.5 \%)$ & $6871(46.8 \%)$ & $<0.001$ \\
\hline Cardiac catheterization & $395(6.2 \%)$ & $504(11.6 \%)$ & $819(20.8 \%)$ & $1718(11.7 \%)$ & $<0.001$ \\
\hline Stress testing & $2008(31.4 \%)$ & $1788(41.2 \%)$ & $1822(46.3 \%)$ & $5618(38.3 \%)$ & $<0.001$ \\
\hline \multicolumn{6}{|c|}{ Physician follow-up 30 days following ED discharge, n (\%) } \\
\hline Cardiology & $1767(27.6 \%)$ & $1525(35.2 \%)$ & $1598(40.6 \%)$ & $4890(33.3 \%)$ & $<0.001$ \\
\hline General/Family physician & $2358(36.8 \%)$ & $1552(35.8 \%)$ & $1474(37.4 \%)$ & $5384(36.7 \%)$ & \\
\hline None & $2279(35.6 \%)$ & $1259(29.0 \%)$ & $864(22.0 \%)$ & $4402(30.0 \%)$ & \\
\hline \multicolumn{6}{|c|}{ All-cause mortality or hospitalization for MI/UA, n (\%) } \\
\hline
\end{tabular}


At 30 days there were 42 deaths $(0.3 \%), 58$ MIs $(0.4 \%), 97$ death/MIs $(0.7 \%)$ and 216 patients that experienced MACE (1.5\%). The NPVs for 30-day MACE was $99.6 \%$ (95\% CI: 99.4 to 99.7 ) for CCS < 2 and $99.7 \%$ (95\% CI: 99.5 to 99.8$)$ for hs-cTn $<5 \mathrm{ng} / \mathrm{L}$. The proportion of patients classified as low risk was higher for the CCS $<2(43.6 \%)$ as compared to hs-cTn $<5 \mathrm{ng} / \mathrm{L}(28.1 \%)$ (difference $=15.5 \%$; $95 \%$ CI: 14.4 to 16.6$)(p<0.001)$. Of the 6404 patients with a CCS $<2$, only $0.4 \%$ had a hs-cTn level $>14 \mathrm{ng} / \mathrm{L}$, while for the 3936 patients with a CCS $>2$, only $1.7 \%$ had a hs-cTn level $<5 \mathrm{ng} / \mathrm{L}$.

The unadjusted and adjusted HRs for the CCS $<2$ were significantly lower for the primary outcome as compared to the CCS $=2$ group (i.e., CCS $<2$ was associated with a $60 \%$ decreased risk of having the composite outcome compared to CCS $=2$ over 30 days) while the CCS $>2$ had significantly higher HRs for the primary outcome (i.e., CCS $>2$ was associated with a 2-fold increased risk of having the composite outcome compared to CCS $=2$ over 30 days) $(p<0.01)$ (Table 2, see Supplement for HRs in Cohort 1 (Table S3) and Cohort 2 (Table S4) for the primary outcome). For the secondary outcomes at 30 days, the adjusted HRs were 0.39 (95\%CI: 0.18 to 0.84 ) for CCS $<2$ and 2.88 (95\%CI: 1.67 to 4.97 ) for CCS $>2$ for death/MI (c-statistic $=0.80)$ and 0.34 (95\%CI: 0.22 to 0.56$)$ for CCS $<2$ and 1.74 (95\%CI: 1.24 to 2.43 ) for CCS $>2$ for MACE (c-statistic $=0.77$ ). Alternatively, setting the CCS $<2$ as the referent group the adjusted HRs for MACE at 30 days for CCS $=2$ was 2.83 (95\%CI: 1.79 to 4.47 ) and for CCS > 2 was 4.91 (95\%CI: 3.04 to 7.91 ) (Table S5). There was a statistically significant difference in the survival probabilities across the CCS groups within 365 days following discharge from ED (Figure 2, log-rank test $p$-value $<0.0001$ for primary outcome, see Supplement for Kaplan-Meier survival curves for all cause death (Figure S1), death/MI (Figure S2), and MACE (Figure S3) with number of patients at risk listed).

Table 2. Cox proportional hazard model estimates for the primary outcome of all-cause mortality, MI, and UA at 30 days, 90 days, and 365 days.

\begin{tabular}{|c|c|c|c|}
\hline Time of Outcome Assessment & Model* & CCS Category & Hazard Ratio (95\% CI) \\
\hline \multirow{6}{*}{30 days } & \multirow[b]{2}{*}{1} & $\mathrm{CCS}<2$ & $0.33(0.19-0.58)$ \\
\hline & & $\mathrm{CCS}>2$ & $2.76(1.89-4.05)$ \\
\hline & \multirow{2}{*}{2} & $\mathrm{CCS}<2$ & $0.38(0.21-0.67)$ \\
\hline & & $\mathrm{CCS}>2$ & $2.31(1.53-3.47)$ \\
\hline & \multirow{2}{*}{3} & $\mathrm{CCS}<2$ & $0.40(0.23-0.72)$ \\
\hline & & $\mathrm{CCS}>2$ & $2.05(1.35-3.13)$ \\
\hline \multirow{6}{*}{90 days } & \multirow{2}{*}{1} & $\mathrm{CCS}<2$ & $0.39(0.25-0.62)$ \\
\hline & & $\mathrm{CCS}>2$ & $4.24(3.09-5.83)$ \\
\hline & \multirow{2}{*}{2} & $\mathrm{CCS}<2$ & $0.49(0.30-0.79)$ \\
\hline & & $\mathrm{CCS}>2$ & $3.26(2.35-4.56)$ \\
\hline & \multirow{2}{*}{3} & $\mathrm{CCS}<2$ & $0.51(0.32-0.82)$ \\
\hline & & $\mathrm{CCS}>2$ & $2.80(1.98-3.97)$ \\
\hline \multirow{6}{*}{365 days } & \multirow{2}{*}{1} & $\mathrm{CCS}<2$ & $0.38(0.29-0.49)$ \\
\hline & & $\mathrm{CCS}>2$ & $3.44(2.85-4.15)$ \\
\hline & \multirow{2}{*}{2} & $\mathrm{CCS}<2$ & $0.56(0.43-0.75)$ \\
\hline & & $\mathrm{CCS}>2$ & $2.20(1.80-2.67)$ \\
\hline & \multirow{2}{*}{3} & $\mathrm{CCS}<2$ & $0.59(0.45-0.78)$ \\
\hline & & $\mathrm{CCS}>2$ & $1.76(1.43-2.17)$ \\
\hline
\end{tabular}

* Model 1 unadjusted; Model 2 adjusted for age and sex; Model 3 adjusted for age, sex, prior history of arrhythmia, heart failure, diabetes, hypertension, MI, peripheral vascular disease, renal disease, stroke and UA. Reference group is CCS $=2$ and all $p$-values are $<0.01$. 


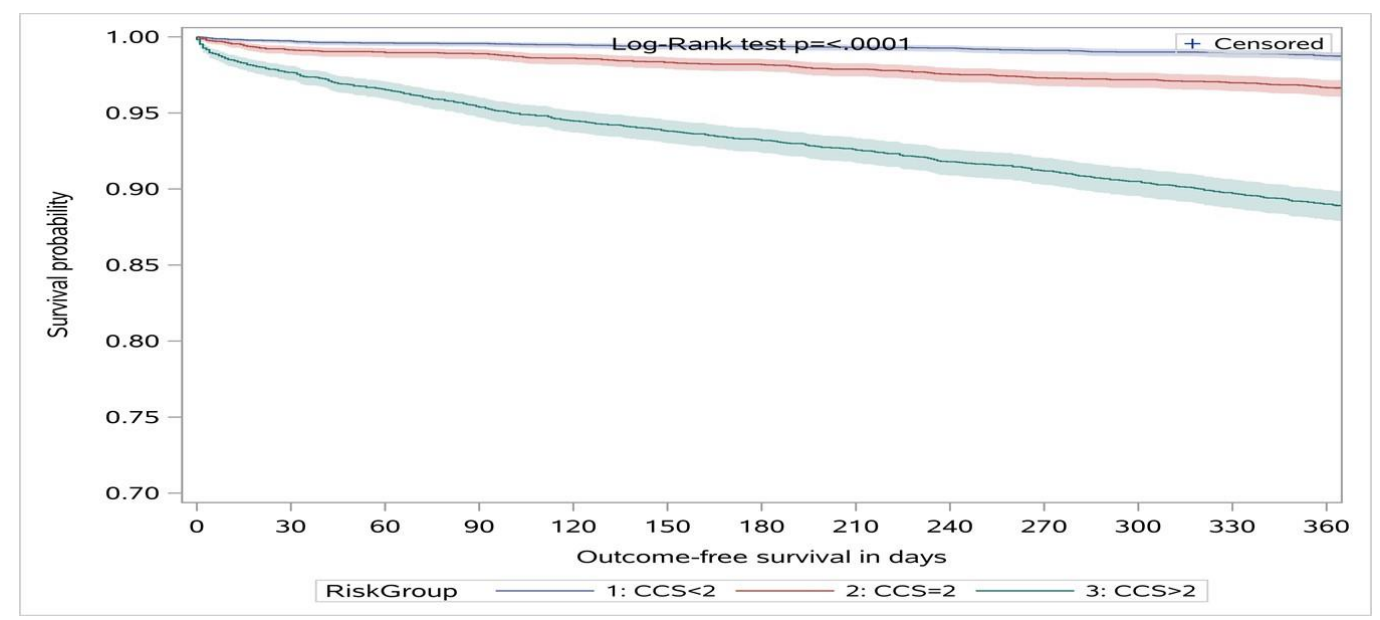

Figure 2. Kaplan-Meier survival curves for the primary outcome (i.e., all-cause death, MI or UA) over 365 days stratified by $\mathrm{CCS}<2, \mathrm{CCS}=2$, and CCS $>2$. CCS: clinical chemistry score.

\section{Discussion}

Patients diagnosed with chest pain and discharged home from the ED in the province of Ontario, Canada have a $1 \%$ composite outcome (all-cause death, MI, UA hospital admission) at one month. This is in agreement with acceptable recommendations from emergency physician groups on miss rates in ED patients with possible ACS symptoms [23,24]. However, within this group of patients, the CCS can further aid in risk stratification. Specifically, a CCS $<2$ represents a group that has nearly half the risk as compared to patients with a CCS $=2$ in experiencing the composite outcome, whereas patients with a CCS $>2$ represent a group that has nearly twice the risk as compared to the CCS $=2$ group. This information and the calculation of the CCS can be readily and easily obtained from laboratory values at ED presentation which may further facilitate decision-making and patient discharge in the ED. Specifically, patients with a CCS $>2$, may benefit from seeing a cardiologist following discharge from the ED to mitigate risk of future adverse events, and incorporation of such a score may affirm the need for appropriate care [27].

Equally important, a greater proportion of the population who are discharged home from the ED can be deemed to be at very low risk when using the CCS $<2(>40 \%)$ as compared to applying a hs-cTn $<5 \mathrm{ng} / \mathrm{L}$ cutoff $(<30 \%)$, thereby reassuring a larger population and possibly avoiding unnecessary medical intervention in these patients. The $5 \mathrm{ng} / \mathrm{L}$ cutoff has been reported as an appropriate cutoff to rule-out for both Roche and Abbott hs-cTn assays [7,8,25]. In this regard, a position paper of the Acute Cardiovascular Care Association which provided an update on the European Society of Cardiology task force report on the management of chest pain provided a management pathway where if the ECG is normal and the hs-cTn is below the limit of detection (e.g., hs-cTnT $<5 \mathrm{ng} / \mathrm{L}$ ), discharge may be appropriate if the patient is pain free, differential diagnoses excluded and the HEART score is $\leq$ 3 [28]. Employing the CCS may further simplify this process and provide additional reassurance in this setting. The CCS may also be used in conjugation with serial measurements of hs-cTn, with the latter being important for assessing acute injury [3,17].

\section{Limitations}

This cohort study has some limitations that merit discussion. First, outcomes were assessed via administrative databases and thus there could be inaccuracies in diagnostic and physician billing codes. However, the approach undertaken here has been validated in other publications with others opining that outcomes via databases provide important real-world data [19-21,29]. Second, as the data are from multiple hospital sites throughout the province of Ontario, there are certainly pre-analytical, analytical and post-analytical variables that may affect high-sensitivity cardiac troponin result reporting and interpretation $[2,9]$. These variables can also affect creatinine and glucose, with a further complication 
being variation due to point of care testing and whole blood measurements [30]. Third, the CCS was retrospectively calculated for each patient and was not used in clinical decision-making. Fourth, we do not have complete information regarding the use of antiplatelet agents, anticoagulants, statins, and beta-blockers amongst the different subgroups of patients (i.e., CCS $<2,2,>2$ ). Fifth, our dataset does not contain information on ejection fraction and the type of stress testing in the study population. Accordingly, a prospective study assessing the CCS in this setting is required.

\section{Conclusions}

Application of the CCS for patients diagnosed with chest pain and discharged home from the ED may further identify low-risk (CCS $<2$ ) and high-risk patients $(C C S>2)$, which may expedite decision-making in the ED and facilitate appropriate post-ED visit clinical care.

Supplementary Materials: The following are available online at http://www.mdpi.com/2077-0383/9/9/2948/s1, Figure S1: Kaplan Meier survival curves for all-cause death over 365 days stratified by CCS $<2, \mathrm{CCS}=2$, and CCS $>2$, Figure S2: Kaplan Meier survival curves for death/MI over 365 days stratified by CCS $<2$, CCS $=2$, and CCS $>2$, Figure S3: Kaplan Meier survival curves for MACE over 365 days stratified by CCS $<2$, CCS $=2$, and CCS $>2$, Table S1: Comparison of baseline characteristics and crude outcomes by CCS $(<2,2,>2)$ for patients with a diagnosis of chest pain discharged home from the ED from Cohort 1, Table S2: Comparison of baseline characteristics and crude outcomes by CCS $(<2,2,>2)$ for patients with a diagnosis of chest pain discharged home from the ED from Cohort 2, Table S3: Cox proportional hazard model estimates for CCS, all-cause mortality plus hospitalization for MI/UA for Cohort 1 with hs-cTnI, Table S4: Cox proportional hazard model estimates for CCS, all-cause mortality plus hospitalization for MI/UA for Cohort 2 with hs-cTnT, Table S5: Cox proportional hazard model estimates (model 3) for the secondary outcome of MACE (defined as the composite of death/MI/UA/PCI or CABG) at 30 days, 90 days, and 365 days with CCS $<2$ as the reference group for the study population $(n=14,676)$.

Author Contributions: Conceptualization, P.A.K.; data curation, P.A.K. and J.O.C.; formal analysis, J.O.C.; funding acquisition, P.A.K.; methodology, P.A.K., D.T.K. and A.W.; project administration, P.A.K. and R.P.; resources, P.A.K. and H.S.; software, J.O.C.; supervision, P.A.K. and A.W.; writing-original draft, P.A.K.; writing一review \& editing, S.E.M., J.S., J.M., B.K.H., H.S., D.T.K. and A.W. All authors have read and agreed to the published version of the manuscript.

Funding: This study was supported by a Canadian Institutes of Health Research grant (PK, funding reference \#155964) with past financial and reagent support by Abbott Laboratories. The funding organizations had no role in the design and conduct of the study; in the collection, analysis, and interpretation of the data, or in the final approval of the manuscript.

Acknowledgments: This study was supported by ICES, which is funded by an annual grant from the Ontario Ministry of Health and Long-Term Care (MOHLTC). Content enclosed are based on data provided by CIHI. Analyses, opinions, and statements expressed herein are those of the authors and not necessarily those of CIHI; no endorsement is intended or should be inferred. We thank IMS Brogan Inc. for use of their Drug Information Database.

Conflicts of Interest: Kavsak has received grants/reagents/consultant/advisor/honoraria from Abbott Laboratories, Abbott Point of Care, Beckman Coulter, Ortho Clinical Diagnostics, Randox Laboratories, Roche Diagnostics and Siemens Healthcare Diagnostics. McMaster University has filed patents with Drs. Kavsak and Worster listed as an inventor in the acute cardiovascular biomarker field, in particular, a patent has been filed on aspects related to the data presented in this study "A Laboratory Score for Risk Stratification for Patients with Possible Cardiac Injury". No other disclosures were reported.

\section{References}

1. Roffi, M.; Patrono, C.; Collet, J.P.; Mueller, C.; Valgimigli, M.; Andreotti, F.; Bax, J.J.; Borger, M.A.; Brotons, C.; Chew, D.P.; et al. 2015 ESC Guidelines for the management of acute coronary syndromes in patients presenting without persistent ST-segment elevation: Task Force for the Management of Acute Coronary Syndromes in Patients Presenting without Persistent ST-Segment Elevation of the European Society of Cardiology (ESC). Eur. Heart J. 2016, 37, 267-315. [PubMed]

2. Wu, A.H.B.; Christenson, R.H.; Greene, D.N.; Jaffe, A.S.; Kavsak, P.A.; Ordonez-Llanos, J.; Apple, F.S. Clinical Laboratory Practice Recommendations for the Use of Cardiac Troponin in Acute Coronary Syndrome: Expert Opinion from the Academy of the American Association for Clinical Chemistry and the Task Force on Clinical Applications of Cardiac Bio-Markers of the International Federation of Clinical Chemistry and Laboratory Medicine. Clin. Chem. 2018, 64, 645-655. [PubMed] 
3. Thygesen, K.; Alpert, J.S.; Jaffe, A.S.; Chaitman, B.R.; Bax, J.J.; Morrow, D.A.; White, H.D.; Executive Group on behalf of the Joint European Society of Cardiology (ESC); American College of Cardiology (ACC); American Heart Association (AHA); et al. Fourth Universal Definition of Myocardial Infarction (2018). Circulation 2018, 138, e618-e651. [CrossRef] [PubMed]

4. Keller, T.; Zeller, T.; Ojeda, F.; Tzikas, S.; Lillpopp, L.; Sinning, C.; Wild, P.; Genth-Zotz, S.; Warnholtz, A.; Giannitsis, E.; et al. Serial changes in highly sensitive troponin I assay and early diagnosis of myocardial infarction. JAMA 2011, 306, 2684-2693. [CrossRef]

5. Kavsak, P.A.; Hill, S.A.; Bhanich Supapol, W.; Devereaux, P.J.; Worster, A. Biomarkers for predicting serious cardiac outcomes at 72 hours in patients presenting early after chest pain onset with symptoms of acute coronary syndromes. Clin. Chem. 2012, 58, 298-302. [CrossRef]

6. Neumann, J.T.; Twerenbold, R.; Ojeda, F.; Sörensen, N.A.; Chapman, A.R.; Shah, A.S.V.; Anand, A.; Boeddinghaus, J.; Nestelberger, T.; Badertscher, P.; et al. Application of High-Sensitivity Troponin in Suspected Myocardial Infarction. N. Engl. J. Med. 2019, 380, 2529-2540. [CrossRef]

7. Chapman, A.R.; Lee, K.K.; McAllister, D.A.; Cullen, L.; Greenslade, J.H.; Parsonage, W.; Worster, A.; Kavsak, P.A.; Blankenberg, S.; Neumann, J.; et al. Association of High-Sensitivity Cardiac Troponin I Concentration With Cardiac Outcomes in Patients With Suspected Acute Coronary Syndrome. JAMA 2017, 318, 1913-1924. [CrossRef]

8. Pickering, J.W.; Than, M.P.; Cullen, L.; Aldous, S.; Ter Avest, E.; Body, R.; Carlton, E.W.; Collinson, P.; Dupuy, A.M.; Ekelund, U.; et al. Rapid Rule-out of Acute Myocardial Infarction With a Single High-Sensitivity Cardiac Troponin T Measurement Below the Limit of Detection: A Collaborative Meta-analysis. Ann. Intern. Med. 2017, 166, 715-724. [CrossRef]

9. Kavsak, P.A. External Quality Assessment Testing Near the Limit of Detection for High-Sensitivity Cardiac Troponin Assays. Clin. Chem. 2018, 64, 1402-1404. [CrossRef]

10. Kavsak, P.A.; Petryayeva, E.; Clark, L. Analytical Variation and Abbott Diagnostics High-Sensitivity Cardiac Troponin I Risk Categories in Asymptomatic Individuals. Can. J. Cardiol. 2019, 35, 1605.e7-1605.e8. [CrossRef]

11. Kavsak, P.A.; Clark, L.; Caruso, N.; Worster, A. Caution When Using High-Sensitivity Cardiac Troponin I Assay to Rule Out Acute Ischemia: When the Delta to Rule In Is Within Analytical Variation. Can. J. Cardiol. 2020, 36, 1161.e11-1161.e12. [CrossRef] [PubMed]

12. Timóteo, A.T.; Papoila, A.L.; Rio, P.; Miranda, F.; Ferreira, M.L.; Ferreira, R.C. Prognostic impact of admission blood glucose for all-cause mortality in patients with acute coronary syndromes: Added value on top of GRACE risk score. Eur. Heart J. Acute Cardiovasc. Care 2014, 3, 257-263. [CrossRef] [PubMed]

13. van Toorenburg, M.; van den Berg, V.J.; van der Ploeg, T.; Heestermans, A.A.; Dirksen, M.T.; Hautvast, R.W.; Drexhage, O.; Boersma, E.; Kardys, I.; Umans, V.A.W.M. Addition of routinely measured blood biomarkers significantly improves GRACE risk stratification in patients with myocardial infarction. Int. J. Cardiol. 2018, 273, 237-242. [CrossRef] [PubMed]

14. Gerbaud, E.; Darier, R.; Montaudon, M.; Beauvieux, M.C.; Coffin-Boutreux, C.; Coste, P.; Douard, H.; Ouattara, A.; Catargi, B. Glycemic Variability Is a Powerful Independent Predictive Factor of Midterm Major Adverse Cardiac Events in Patients With Diabetes With Acute Coronary Syndrome. Diabetes Care 2019, 42, 674-681. [CrossRef]

15. Kavsak, P.A.; Neumann, J.T.; Cullen, L.; Than, M.; Shortt, C.; Greenslade, J.H.; Pickering, J.W.; Ojeda, F.; Ma, J.; Clayton, N.; et al. Clinical chemistry score versus high-sensitivity cardiac troponin I and T tests alone to identify patients at low or high risk for myocardial infarction or death at presentation to the emergency department. CMAJ 2018, 190, E974-E984. [CrossRef]

16. Kavsak, P.A.; McRae, A.; Vatanpour, S.; Ismail, O.Z.; Worster, A. A Multicenter Assessment of the Sensitivity and Specificity for a Single High-Sensitivity Cardiac Troponin Test at Emergency Department Presentation for Hospital Admission. J. Appl. Lab. Med. 2019, 4, 170-179. [CrossRef]

17. Kavsak, P.A.; Mondoux, S.E.; Ma, J.; Sherbino, J.; Hill, S.A.; Clayton, N.; Mehta, S.R.; Griffith, L.E.; McQueen, M.; Devereaux, P.J.; et al. Comparison of two biomarker only algorithms for early risk stratification in patients with suspected acute coronary syndrome. Int. J. Cardiol. 2020. [CrossRef]

18. Kavsak, P.A.; Cerasuolo, J.O.; Ko, D.T.; Ma, J.; Sherbino, J.; Mondoux, S.E.; Clayton, N.; Hill, S.A.; McQueen, M.; Griffith, L.E.; et al. Using the Clinical Chemistry Score in the emergency department to detect adverse cardiac events: A diagnostic accuracy study. CMAJ Open 2020, accepted. 
19. Lau, G.; Koh, M.; Kavsak, P.A.; Schull, M.J.; Armstrong, D.W.J.; Udell, J.A.; Austin, P.C.; Wang, X.; Ko, D.T. Clinical outcomes for chest pain patients discharged home from emergency departments using high-sensitivity versus conventional cardiac troponin assays. Am. Heart J. 2020, 221, 84-94. [CrossRef]

20. Kavsak, P.A.; Cerasuolo, J.O.; Ko, D.T.; Ma, J.; Sherbino, J.; Mondoux, S.E.; Perez, R.; Seow, H.; Worster, A. High-Sensitivity Cardiac Troponin I vs a Clinical Chemistry Score for Predicting All-Cause Mortality in an Emergency Department Population. CJC Open 2020, 2, 296-302. [CrossRef]

21. Kavsak, P.A.; Cerasuolo, J.O.; Ko, D.T.; Perez, R.; Seow, H.; Ma, J.; Worster, A. High-Sensitivity Cardiac Troponin T Testing and Cardiovascular Outcomes at 30 Days and 1 Year in Patients Discharged Home from the Emergency Department with Chest Pain. J. Appl. Lab. Med. 2020, 5, 821-824. [CrossRef] [PubMed]

22. Levey, A.S.; Stevens, L.A.; Schmid, C.H.; Zhang, Y.L.; Castro, A.F., 3rd; Feldman, H.I.; Kusek, J.W.; Eggers, P.; Van Lente, F.; Greene, T.; et al. A new equation to estimate glomerular filtration rate. Ann. Intern. Med. 2009, 150, 604-612. [CrossRef] [PubMed]

23. Than, M.; Herbert, M.; Flaws, D.; Cullen, L.; Hess, E.; Hollander, J.E.; Diercks, D.; Ardagh, M.W.; Kline, J.A.; Munro, Z.; et al. What is an acceptable risk of major adverse cardiac event in chest pain patients soon after discharge from the Emergency Department? A clinical survey. Int. J. Cardiol. 2013, 166, 752-754. [CrossRef] [PubMed]

24. Tomaszewski, C.A.; Nestler, D.; Shah, K.H.; Sudhir, A.; Brown, M.D. Clinical Policy: Critical Issues in the Evaluation and Management of Emergency Department Patients with Suspected Non-ST-Elevation Acute Coronary Syndromes. Ann. Emerg. Med. 2018, 72, e65-e106. [CrossRef] [PubMed]

25. Eggers, K.M.; Jernberg, T.; Ljung, L.; Lindahl, B. High-Sensitivity Cardiac Troponin-Based Strategies for the Assessment of Chest Pain Patients-A Review of Validation and Clinical Implementation Studies. Clin. Chem. 2018, 64, 1572-1585. [CrossRef]

26. Than, M.P.; Pickering, J.W.; Sandoval, Y.; Shah, A.S.V.; Tsanas, A.; Apple, F.S.; Blankenberg, S.; Cullen, L.; Mueller, C.; Neumann, J.T.; et al. Machine Learning to Predict the Likelihood of Acute Myocardial Infarction. Circulation 2019, 140, 899-909. [CrossRef]

27. Wong, M.K.; Wang, J.T.; Czarnecki, A.; Koh, M.; Tu, J.V.; Schull, M.J.; Wijeysundera, H.C.; Lau, C.; Ko, D.T. Factors associated with physician follow-up among patients with chest pain discharged from the emergency department. CMAJ 2015, 187, E160-E168. [CrossRef]

28. Stepinska, J.; Lettino, M.; Ahrens, I.; Bueno, H.; Garcia-Castrillo, L.; Khoury, A.; Lancellotti, P.; Mueller, C.; Muenzel, T.; Oleksiak, A.; et al. Diagnosis and risk stratification of chest pain patients in the emergency department: Focus on acute coronary syndromes. A position paper of the Acute Cardiovascular Care Association. Eur. Heart J. Acute Cardiovasc. Care. 2020, 9, 76-89. [CrossRef]

29. Meah, M.N.; Denvir, M.A.; Mills, N.L.; Norrie, J.; Newby, D.E. Clinical endpoint adjudication. Lancet 2020, 395, 1878-1882. [CrossRef]

30. Kavsak, P.A.; Zielinski, N.; Li, D.; McNamara, P.J.; Adeli, K. Challenges of implementing point-of-care testing (POCT) glucose meters in a pediatric acute care setting. Clin. Biochem. 2004, 37, 811-817. [CrossRef] 